\title{
Prevalencia y factores asociados al traumatismo craneoencefálico en adultos entre 20 a 50 años que acuden al servicio de emergencia del hospital Vicente Corral Moscoso, periodo enero-junio 2018
}

\section{Prevalence and factors associated with cranioencephalic trauma in adults between 20 and 50 years of age attending the emergency service of the Vicente Corral Moscoso hospital, January-June 2018}

\author{
María Castro Pomaquiza ${ }^{1}$, Pablo Lazo Pillaga ${ }^{1}$, Johnny Vizuela Carpio ${ }^{1}$, María José Serrano Serrano ${ }^{1}$, Nube \\ Pacurucu-Avila*1 \\ ${ }^{1}$ Universidad Católica de Cuenca, Ecuador \\ *npacurucua@ucacue.edu.ec
}

DOI: https://doi.org/10.26871/killkanasalud.v4i4.747

\begin{abstract}
Resumen
Objetivo: Identificar la prevalencia y factores asociados al traumatismo craneoencefálico en adultos entre 20 a 50 años que acuden al Servicio de Emergencia del Hospital Vicente Corral Moscoso, periodo Enero - Junio 2018. Metodología: Estudio retrospectivo, cuantitativo y descriptivo de corte transversal, el universo está constituido por 135 pacientes atendidos en el Hospital Vicente Corral Moscoso en el área de emergencia con trauma craneoencefálico, de los cuales se analizaron 100 historias clínicas de pacientes que cumplieron los criterios de inclusión. El instrumento para recolectar datos fue un formulario previamente validado de 9 ítems: las 5 primeras determinaron las variables sociodemográficas, y en los 4 consiguientes se determinaron la gravedad de trauma, mecanismo de trauma, y condiciones del paciente, y para el análisis de los mismos se utilizó el programa Statisyical Product and Service Solutions para Windows. Resultados: El $90 \%$ correspondiendo al trauma leve. El grupo etario más afectado fue de 20-30 años, predominando el sexo masculino en un $71 \%$, el nivel de instrucción de los pacientes analizados fue secundario $42 \%$, estado civil soltero(a) $44 \%$, con mayor predominio en pacientes de condición socioeconómica media $77 \%$, la causa principal del traumatismo craneoencefálico fue los accidentes de tránsito $34 \%$, y fallecieron el $1 \%$ de la población estudiada. Conclusión: La frecuencia de traumatismo craneoencefálico es un problema de salud elevado en el contexto epidemiológico en el Hospital Vicente Corral Moscoso de la ciudad de Cuenca, por eso es necesario capacitar a toda la población sobre cuidados al momento de realizar actividades y la utilización de medidas de protección respectivas según la actividad que realicen.
\end{abstract}

Palabras clave: traumatismo craneoencefálico, lesiones encefálicas, escala de coma de Glasgow, urgencias médicas.

\section{Abstract}

Objective: To identify the prevalence and factors associated with brain injuries in adults between 20 to 50 years old who attend the emergency service of the Vicente Corral Moscoso Hospital, period January - June 2018. Methodology: Retrospective, quantitative and descriptive cross-sectional study, the universe consists of 135 patients treated at Vicente Corral Moscoso Hospital in the emergency area with head trauma, of which 100 clinical histories of patients who met the inclusion criteria were analyzed. The data collection instrument was a previously validated form of 9-items: the first 5 determined sociodemographic variable and the resulting 4 determined the severity of trauma, trauma mechanism, and patient conditions, and the Statistical Product and Service Solutions for Windows was used for their analysis. Results: $90 \%$ corresponding to mild trauma. The most affected group was 20-30 years old, predominantly male in $71 \%$, the level of instruction of the patients tested was secondary $42 \%$, single marital status $44 \%$ with the highest predominance in patients with an average socioeconomic condition of $77 \%$ and the most predominant socioeconomic conditions were the average 77\%, the main cause of head trauma was 34\% traffic accidents, and 1\% of the studied population died. Conclusion: The frequency of head trauma is a high health problem in the epidemiological context at the Vicente Corral Moscoso Hospital in the city of Cuenca, that's why it's necessary to train the entire population on care when doing activities and the use of respective protective measures depending on their activity.

Keywords: traumatic, brain injuries, Glasgow coma scale, emergencies . 


\section{Introducción}

El trauma craneoencefálico se define como una alteración en la función cerebral u otra evidencia de patología del cerebro, causada por una fuerza externa. Según la Organización Mundial de la Salud (OMS), es considerada a nivel mundial como un grave problema de salud pública, ocasionando más de 5 millones de muertes en el año, una cifra igual a la muerte por VIH/SIDA. ${ }^{1}$

Debido a los traumatismos, en la actualidad han sido afectadas más de 5,8 millones de personas. Según reportes del Instituto Nacional de Estadística y Censo (INEC), en Ecuador en el año 2015 se notificaron 106,751 ingresos hospitalarios por traumatismos. En la ciudad de Cuenca por la misma causa fueron ingresados un total de 70,745 hombres y 36,006 mujeres. ${ }^{2,3}$ En España la incidencia de traumatismo craneoencefálico es de 200 casos por cada 100 mil habitantes, en los Estados Unidos cada año 1,1 millones de pacientes entre los 25 a 35 años de edad son tratados en la sala de emergencia por traumatismo craneoencefálico, en México, el traumatismo craneoencefálico es la tercera causa de muerte, con 35,567 defunciones; un índice de mortalidad de 38.8 por 100 .mil habitantes. ${ }^{4-6}$

Más del $90 \%$ de muertes por traumatismo se producen en países con ingresos medios y bajos, debido a que la financiación de investigaciones es muy insuficiente y no se han asignado recursos suficientes para fortalecer la prestación de servicios médicos en los centros de salud para afrontar este problema. ${ }^{7}$

Debido al creciente número de pacientes con traumatismo se ha visto la necesidad de realizar el estudio de traumatismo craneoencefálico y sus factores asociados en adultos de 20 a 50 años del Hospital Vicente Corral Moscoso de la ciudad de Cuenca, cuyos resultados obtenidos servirán de base para posteriores investigaciones institucionales y no institucionales.

\section{Materiales y metodos}

Estudio retrospectivo, cuantitativo y descriptivo de corte transversal, se llevó a cabo a través del análisis de historias clínicas de pacientes con edades entre 20 y 50 años con traumatismo craneoencefálico que fueron atendidos en el servicio de emergencia del Hospital Vicente Corral Moscoso durante el año 2018. Se excluyó a todos los pacientes pediátricos, adolescentes y adultos mayores y aquellos que abandonaron el servicio de emergencia antes del registro completo de la información o sin previa alta médica.

El tamaño de la muestra se obtuvo mediante la utilización de la fórmula de la unidad de epidemiologia clínica y bioestadística, de una población de 135 pacientes tanto hombres como mujeres atendidos, con un nivel de confianza del $95 \%$ y precisión del $5 \%$, obteniéndose un total de 100 participantes que fueron atendidos en el Servicio de Emergencia del Hospital Vicente Corral Moscoso.

El plan metodológico con el que se realizó la investigación fue de tipo deductivo, mediante la técnica de revisión de documento, y el instrumento fue una ficha de recolección de datos. Previa a la investigación se planteó el protocolo que fue presentado y autorizado por el Comité de Bioética e Investigación en Seres Vivos de la Universidad Católica de Cuenca. Además, se solicitó la autorización pertinente para la aplicación del instrumento de recopilación de datos mediante un consentimiento informado dirigido a la comisión de docencia e investigación del Hospital Vicente Corral Moscoso. No fue necesaria la autorización de los pacientes participantes debido que la información para este proyecto se obtendrá mediante la revisión de historias clínicas.

Una vez obtenida la autorización del hospital se revisó una por una las historias clínicas de pacientes con edades comprendidas entre 20 y 50 años que presentaban traumatismos craneoencefálico, los datos del estudio se registraron manualmente en un formulario que fue validado por tres expertos y la confiabilidad se realizó mediante Alfa de Cronbach con un resultado de 0,807 , el mismo constaba de 9 ítems, las 5 primeras determinaron las variables sociodemográficas, y en los 4 consiguientes se determinaron la gravedad de trauma, mecanismo de trauma, y condiciones del paciente.

Una vez recolectada la información se procedió a organizarla para luego digitalizarla en el programa estadístico SPSS (Statisyical Product and Service Solutions para Windows), finalmente se obtuvieron los resultados para cada uno de los ítems de las variables que se plantearon representados en tablas con frecuencias y porcentajes.

\section{Resultados}

Los resultados obtenidos de las 100 historias clínicas de pacientes con traumatismo craneoencefálico atendidos en el servicio de emergencia del Hospital Vicente Corral Moscoso demuestran que dentro de las características sociodemográficas existe un predominio en el sexo masculino en edades entre 20 y 30 años, de estado civil soltero, con nivel de instrucción secundaria y de condición socioeconómica media, que habitan dentro del casco urbano. (Tabla 1). De acuerdo a la distribución de los mecanismos de trauma tenemos en primer lugar aquellos causados por los accidentes de tránsito (34\%), seguido de las caídas (32\%), otros; como violencia, accidentes por armas de fuego, armas blancas, etc. $(30 \%)$ y finalmente los accidentes laborales (4\%). (Tabla 2). Según la clasificación del grado de severidad del traumatismo se utilizó como guía la escala de Glasgow en donde se observa mayor frecuencia en traumatismo leve (90\%). (Tabla 3). En la Tabla 4 se identifica los resultados de las condiciones del paciente luego de haberle brindado cuidados asistenciales en el servicio de emergencia en el cual se obtuvo que de las 100 historias revisadas fallecieron el $1 \%$ de los atendidos. 
Tabla 1: Características sociodemográficas en los adultos de 20 a 50 años con traumatismo craneoencefálico en el Hospital Vicente Corral Moscoso

\begin{tabular}{|c|c|c|}
\hline VARIABLE & $\begin{array}{l}\text { FRECUENCIA } \\
\text { EDAD }\end{array}$ & PORCENTAJE \\
\hline $20-30$ & 45 & $45 \%$ \\
\hline $30-40$ & 38 & $38 \%$ \\
\hline $40-50$ & 17 & $17 \%$ \\
\hline \multirow[t]{2}{*}{ Total } & 100 & $100 \%$ \\
\hline & SEXO & \\
\hline Masculino & $71 *$ & $71 \%$ \\
\hline Femenino & 29 & $29 \%$ \\
\hline \multirow[t]{2}{*}{ Total } & 100 & $100 \%$ \\
\hline & \multicolumn{2}{|c|}{ NIVEL DE INSTRUCCIÓN } \\
\hline Primaria & 39 & $39 \%$ \\
\hline Secundaria & 42 & $42 \%$ \\
\hline Superior & 15 & $15 \%$ \\
\hline Ninguno & 4 & $4 \%$ \\
\hline \multirow[t]{2}{*}{ Total } & 100 & $100 \%$ \\
\hline & \multicolumn{2}{|l|}{ ESTADO CTVIL } \\
\hline soltera(o) & 44 & $44 \%$ \\
\hline $\operatorname{casada}(0)$ & 36 & $36 \%$ \\
\hline divorciada(o) & 8 & $8 \%$ \\
\hline unión libre & 12 & $12 \%$ \\
\hline Total & 100 & $100 \%$ \\
\hline \multicolumn{3}{|c|}{ PROCEDENCIA } \\
\hline Rural & 29 & $29 \%$ \\
\hline Urbano & $71^{*}$ & $71 \%$ \\
\hline \multirow[t]{2}{*}{ Total } & 100 & $100 \%$ \\
\hline & \multicolumn{2}{|c|}{ CONDICIONES SOCIO ECONÓMICAS } \\
\hline Bajo & 20 & $20 \%$ \\
\hline Medio & 77 & $77 \%$ \\
\hline Alto & 3 & $3 \%$ \\
\hline Total & 100 & $100 \%$ \\
\hline
\end{tabular}

Tabla 2: Distribución de los mecanismos que causan el traumatismo craneoencefálico

\begin{tabular}{lcc}
\hline \multicolumn{1}{c}{ MECANISMO DE TRAUMA } & FRECUENCIA & PORCENTAJE \\
\hline Accidente de transito & 34 & 34 \\
Caída & 32 & 32 \\
Accidente laboral & 4 & 4 \\
Otros (violencias, maltrato, accidentes por & 30 & 30 \\
arma de fuego arma blanca, etc.) & & \\
Total & $\mathbf{1 0 0}$ & $\mathbf{1 0 0} \%$ \\
\hline
\end{tabular}

Tabla 3: Clasificación del grado de severidad del traumatismo craneoencefálico

\begin{tabular}{lrr}
\hline $\begin{array}{c}\text { GRADO DE SEVERIDAD DEL } \\
\text { TRAUMA }\end{array}$ & FRECUENCIA & PORCENTAJE \\
\hline Traumatismo leve & 90 & 90 \\
Traumatismo moderado & 6 & 6 \\
Traumatismo grave & 4 & 4 \\
Total & 100 & $\mathbf{1 0 0 \%}$ \\
\hline
\end{tabular}

Tabla 4: Identificación de las condiciones del paciente luego de brindar cuidados asistenciales

\begin{tabular}{lrr}
\hline CONDICIÓN DEL PACIENTE & FRECUENCIA & PORCENTAJE \\
\hline Vivo & 99 & 99 \\
Muerto & 1 & 1 \\
Total & 100 & $100 \%$ \\
\hline
\end{tabular}

\section{Discusión}

En este estudio se encontró que el traumatismo craneoencefálico es un problema de salud muy elevado en el contexto epidemiológico en el Hospital Vicente Corral Moscoso de la ciudad de Cuenca. Al analizar los factores asociados a estos traumatismos se pudo observar una mayor prevalencia en el sexo masculino $(71 \%)$, Los resultados obtenidos coinciden con el estudio de Herrera ${ }^{8}$ en Brasil en el año 2018 donde reveló que el $83 \%$ de los afectados son de sexo masculino. Petgrave ${ }^{9}$ en Costa Rica en el año 2016, también indico que el sexo masculino registró la mayoría de los casos. Pérez ${ }^{10}$ en Cuba en el año 2020 en su estudio reveló un predomino del $79,2 \%$ en el sexo masculino. Otro estudio con el que coincide es con el de Ortega ${ }^{11}$ en España en el año 2018 que muestra mayor frecuencia de traumatismos en el sexo masculino, Rondón ${ }^{12}$ en Perú el año 2016 dio a conocer que existe un predominio en el sexo masculino $(68,47 \%)$ a diferencia del sexo femenino $(31,53 \%)$, No se ha encontrado ningún estudio que discrepe con los resultados obtenidos.

En cuanto a la edad los resultados indican predominio en el grupo etario entre 20 y 30 años, que tiene similitud con el estudio de Herrera ${ }^{8}$ en Brasil en el año 2018 que indica que el grupo de edad más frecuente fue entre $21 \mathrm{y}$ 30 años y con la investigación de Ortega ${ }^{11}$ en el año 2018 en España donde predomina las edades entre 20 y 35 años con el 52,2\%. Los resultados obtenidos discrepan con los estudios de Petgrave ${ }^{9}$ en Costa Rica en el 2016 y Piña ${ }^{13}$ en el año 2012 en Cuba que señalan un predominio en los mayores de 43 años (54\%) y mayores de 45 años (56\%) respectivamente. También con el estudio de González ${ }^{14}$ en España en el año 2015 donde indica que personas de 45 años tienen más posibilidad de sufrir traumatismo craneoencefálico.

Los resultados indican que el traumatismo craneoencefálico prevalece en pacientes con estudios secundarios de estado civil soltero y de condición socioeconómica media, que tiene similitud con los resultados del estudio de Flores ${ }^{1}$ en el año 2018 en Perú que señala que los pacientes tenían un grado de instrucción secundaria o superior completa y que contaban con empleo, pero discrepa con el estudio de Ortega $^{11}$ en España en el año 2018 y el de Pari ${ }^{15}$ en el año 2018 en Perú donde indica que los pacientes tenían un grado de instrucción primaria, de estado civil casado y de condición socioeconómica bajo.

En relación al mecanismo del trauma existe predomino en aquellos causado por accidentes de tránsito, que se asemeja con los estudios de Petgrave ${ }^{9}$ en el año 2016 en Costa Rica, González ${ }^{14}$ en España en el año 2015, Cunha ${ }^{16}$ en Brasil en el año 2018, y Bermúdez ${ }^{17}$ en Cuba en el año 2020 donde también indican una prevalencia de traumas en accidentes de tránsito. Lo que discrepa con los estudios de Flores $^{1}$ en Perú en el año 2018, Ortega ${ }^{11}$ en el año 2018 en España, Rodríguez ${ }^{18}$ en Cuba en el año 2020 y Paredes ${ }^{19}$ en Ecuador en el año 2020 donde revelan que los 
mecanismos causantes del trauma craneoencefálico tienen mayor incidencia por caídas ya sea laborales o domésticas.

Según el grado de severidad del trauma se analizó mayor frecuencia en traumatismo leve, lo que coincide con el estudio de Pari ${ }^{15}$ en Perú en el año 2018 y Cunha $^{16}$ en el año 2018 en Brasil que también señala el trauma leve con mayor prevalencia $(41,29 \%)$ y $(32,5 \%)$ respectivamente, pero discrepan con los estudios de Rondón ${ }^{12}$ en Perú en el año 2016, Paredes ${ }^{19}$ en Ecuador en el año 2020 y Suárez ${ }^{20}$ en Cuba en el año 2017 que como resultado obtuvieron mayor incidencia en traumas graves todos guiados según la escala de Glasgow.

Según la condición de paciente fue favorable gracias a los cuidados asistenciales que recibieron dentro del área de emergencia, los resultados tienen similitud con las investigaciones de Ortega ${ }^{11}$ en España en el año 2018, Paredes ${ }^{19}$ en Ecuador en el año 2020 y Amado $^{20}$ en Cuba en el año 2017 en donde los pacientes que presentaban traumas leves y fueron atendidos de manera inmediata no presentaron alteraciones en su salud, lo que discrepa con los estudios de Pérez ${ }^{10}$ en el año 2020 en Cuba y Piña ${ }^{13}$ en Cuba en el año 2012 que se obtuvo como resultado que por más que se brindó la atención necesaria en el área de emergencia los pacientes acudían con lesiones graves y por ende causaban la muerte.

\section{Conclusion}

Se encontró una alta prevalencia de traumatismo craneoencefálico en el Hospital Vicente Corral Moscoso de la ciudad de Cuenca, en el periodo de Enero-Junio 2018, principalmente en el sexo masculino en edades comprendidas entre 20 y 30 años de estado civil soltero, con nivel de instrucción secundaria y de condición socioeconómica media. La principal causa de trauma fueron los accidentes de tránsito con grado de severidad leve y excelentes condiciones del paciente al ser atendidos en el servicio de emergencia. Por estas razones antes mencionadas es importante dar a conocer estos resultados para capacitar a toda la población sobre cuidados al momento de realizar actividades y la utilización de medidas de protección respectivas según la actividad que realicen.

\section{Fuente de Financiamiento}

Este estudio es autofinanciado.

\section{Conflicto de Intereses}

No existen conflictos personales, profesionales, financie-12. Rondón MA. "Características epidemiológicas del trauroso de otro tipo.

\section{Consentimiento Informado}

Los autores cuentan con el consentimiento informado de los pacientes para la investigación, la publicación del caso 13 . y sus imágenes.

\section{Referencias Bibliográficas}

1. Flores- Botton L. Factores pre quirúrgicos asociados a mortalidad en pacientes sometidos a craniectomía descomprensiva por traumatismo craneoencefálico grave. (tesis para título de médico cirujano). Trujillo-Perú. Universidad Privada Antenor Orrego. 2018.

2. INEC. Registro estadístico de camas y egresos hospitalarios. Gestión de Estadísticas Sociodemográficas en Base a Registros Administrativos. Quito Ecuador. 2018.

3. Astudillo- Molina R, Salamea Molina JC, Crespo- Riquetti P, Salamea Molina P. Trauma, Diez años de experiencia Hospital Vicente Corral Moscoso. Rev. Fac Cien Med. 2010; 29(31):23-30. DOI: 10.13140/2.1.2752.4809

4. Amado A, Cañizares O, Alba L, Alegret M. Caracterización epidemiológica y neurológica del traumatismo craneoencefálico frontal durante cinco años en Villa Clara. Medicentro (Villa Clara). 2017; 21(1).

5. Aleman R, Widmark B. Incidencia del traumatismo craneoencefálico en usuarios que acudieron al "Hospital San Vicente de Paul" en el periodo 2015-2017. (tesis para título de licenciado en enfermería). Ibarra-Ecuador Universidad Técnica del Norte. 2019.

6. Muñana- Rodríguez JE, Ramírez- Elías A. Escala de coma de Glasgow: origen, análisis y uso apropiado. Enferm. Univ. 2014; 11(1):24-35. DOI: 10.1016/S16657063(14)72661-2

7. Amado A, Cañizares O, Alba L, Alegret M. Caracterización neuropsicológica de pacientes con trauma craneoencefálico frontal leve y moderado. Acta Médica del Centro. 2017; 11(2).

8. Herrera M, Ariza A, Rodríguez J, Pacheco A. Epidemiologia del trauma craneoencefálico. Rev. Cubana de medicina intensiva y emergencias. 2018; 17(2):3-6.

9. Petgrave A, Padilla J, Díaz J, Chacón R, Chaves C, et al. Perfil Epidemiológico del traumatismo craneoencefálico en el servicio de Neurocirugía de Hospital Dr. Rafael A. Calderón Guardia durante el periodo 2007 al 2012. SENEC. 2016; 27(3):112-120. https://doi.org/10.1016/j.neucir.2015.10.003

10. Pérez-Pérez RM, Linares-Cánovas LP, GonzálezHernández A, Romero-Valdés YC, Rodríguez-Villalonga OL. Comportamiento del trauma craneoencefálico en el Hospital General Docente "Abel Santamaría Cuadrado". Univ Med Pinareña. 2020; 16(1); e438.

11. Ortega JM, Lomillos N, Choque B, Tamarit M, Poveda P, Remedios M, et al. Traumatismo craneoencefálico leve. Surg Neurol Int.2018; 9(1): S16-S18. DOI: 10.4103/sni.sni_371_17 matismo encéfalo craneano por accidente de tránsito en el Hospital Regional Honorio Delgado de Arequipa julio 2014 a junio 2015". (tesis para título de médico cirujano). Arequipa- Perú. Universidad Católica de Santa María de Arequipa. 2016.

. Piña-Tornes A, Garcés-Hernández R, VelázquezGonzález E, Lemes-Báez JJ. Factores pronósticos en 
el traumatismo craneoencefálico grave del adulto. Rev Cubana Neurol Neurocir. 2012; 2(1): 28-33.

14. Gonzalez-Robledo J, Martin-González F, Moreno-García M, Sánchez-Barba M, Sánchez -Hernández F. Factores pronósticos relacionados con la mortalidad de paciente con trauma grave: desde la atención prehospitalaria hasta la Unidad de Cuidados Intensivos. Med. Intensiva. 2015; 39(7): 412-421. DOI: 10.1016/j.medin.2014.06.004

15. Pari-Tola V. "Factores asociados a la severidad del traumatismo craneoencefálico en adultos internados en el Hospital Regional Honorio Delgado- Arequipa 2017”. (tesis para título de médico cirujano.). Juliaca-Perú. Universidad Andina "Néstor Cáceres Velázquez". 2018.

16. Cunha-Vasconcelos A, Capistrano-Dias J, Plegge-Ristow M, Andrade-Vieira W, et al. Epidemiological Aspects of Cranioencephalic Trauma at Cuiabá Municipal Hospital, Brazil. Int.J. Odontostomat.2018;12(1): 29-34.

17. Bermúdez-Ruíz JA, Merlán-Martínez M, Vitón-Castillo AA, Brunet-Liste JV, Lara-Merlan LM. Caracterización de pacientes con traumatismo craneoencefálico severo atendidos en el Hospital General Docente "Arnaldo Milián Castro”. Univ Med Pinareña. 2020;16(1): e376.

18. Rodríguez-Venegas EC, Chirino-Chiang AA, FontaineOrtiz JE, Hernández-García OL, Zamora-Fung R. Traumatismo craneoencefálico en pacientes atendidos en el Hospital Militar Central "Dr. Carlos Juan Finlay". Univ Med Pinareña. 2020;16(1): e375.

19. Paredes- Zambrano K, Cedeño-Veintimilla MS, De los Ríos-Tomalá PG, Vaca-Morla F. Factores de riesgo y complicaciones del traumatismo craneoencefálico en adulto joven. RECIMUNDO. 2020;142-151. DOI: 10.26820/recimundo/4.

20. Suarez-Monnet D, Medrano-García R, López-Delgado H. Factores pronósticos del trauma craneoencefálico moderado. Comportamiento en un periodo de un año. Rev. Archivo Médico de Camagüey.2017;10(3): 38-50.

Recibido: 10 de octubre de 2020

Aceptado: 27 de octubre de 2020

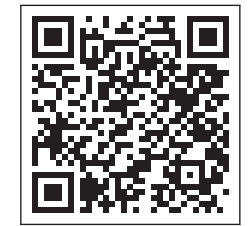


